\title{
Romanian National Guideline on Translating Fecal Microbiota Transplantation Applications related to Clostridioides difficile Infections into the Local Clinical Practice
}

\author{
Georgiana-Emmanuela Gîlcă-Blanariu' ${ }^{1}$, Gabriela Ștefănescu1 ${ }^{1}$, Irina Gîrleanu ${ }^{1}$, Tariq Iqbal ${ }^{2}$, Jonathan Segal ${ }^{3}$, Benjamin \\ Mullish $^{4}$, Mohammed Nabil Quraishi' ${ }^{2}$, Josbert Keller ${ }^{5,6}$, Tamas Molnar ${ }^{7}$ Francis Megraud ${ }^{8}$, Dan Dumitrașcu ${ }^{9}$, Mircea \\ Manuc $^{10}$, Lumința Samaranda Iancu ${ }^{11}$, Cristina Marica ${ }^{9}$, Cristian Gheorghe ${ }^{10}$, Susan Manzoor ${ }^{2}$, Anca Trifan ${ }^{1}$
}

1) Grigore T. Popa Univ. of

Medicine and Pharmacy, Institute

of Gastroenterol and Hepatol,

St. Spiridon Univ. Hospital,

Iași, Romania; 2) University

of Birmingham, Birmingham,

United Kingdom; 3) Hillingdon

Hospital London, London,

United Kingdom; 4) Imperial

College London, London,

United Kingdom; 5) Depart.

of Gastroenterol. Haaglanden

Medical Center, The Hague,

The Netherlands; 6) Leiden

Univ. Medical Center, Leiden,

The Netherlands; 7) Depart.

of Internal Medicine, Univ. of

Szeged, Szeged, Hungary; 8)

Univ. of Bordeaux, Bordeaux,

France; 9) Iuliu Hatieganu Univ.

of Medicine and Pharmacy, $2^{\text {nd }}$

Depart. of Internal Medicine,

Cluj-Napoca, Romania; 10) Carol

Davila Univ. of Medicine and

Pharmacy, Digestive Diseases

and Liver Transplantation Center,

Fundeni Clinical Institute,

Bucharest, Romania; 11) Grigore

T. Popa Univ. of Medicine

and Pharmacy, Depart. of

Microbiology, Iași, Romania;

\section{Address for correspondence: Irina Gîrleanu, MD, PhD, Depart. of Gastroenterol, Gr. T. Popa Univ of Medicine and Pharmacy, Iași, Romania; gilda_iri25@ yahoo.com}

Received: 07.12.2020

Accepted: 15.01.2021

*Society of Gastroenterology and Hepatology

\section{ABSTRACT}

Fecal microbiota transplantation involves the infusion of intestinal microorganisms via the transfer of a stool from a healthy individual into a diseased individual, with the intent of restoring normal intestinal flora. Fecal transplant is proposed for the treatment of refractory Clostridioides difficile infection. At present, recurrent Clostridioides difficile infection is the only indication supported by solid scientific evidence. Regulations by healthcare authorities vary among different countries. Considering that Romania does not have an available national guideline to offer standardization, this paper aimed to create a national fecal microbiota transplantation guideline concerning indications, techniques and donor screening, developed by international and local scientific working groups.

Key words: Clostridioides difficile infection - dysbiosis - fecal microbiota transplantation - guideline-stool transplantation.

Abbreviations: BMI: body mass index; CD: Clostridioides difficile; CDI: Clostridioides difficile infection; ESBL: extended spectrum beta-lactamase; FDA: Food Drug Administration; FMT: fecal microbiota transplantation; GI: gastrointestinal; GRADE: Grading of Recommendations Assessment, Development and Evaluation; ICU: intensive care unit; MDRD: multi-drug resistant organisms; mTOR: mammalian target of rapamycin; MELD: Model for End-Stage Liver Disease; MRSA: Methicilin-resistant Staphyloccocus aureus; NG: naso-gastric; PPI: proton pump inhibitors; rCDI: recurrent Clostridioides difficile infection; RCT: randomized controlled trial; UC: ulcerative colitis; SOT: solid organ transplant; TPHA: Trepomena pallidum antibodies; VRE: vancomycin rezistent enterococci; WG: working group.

\section{RATIONALE OF THE NATIONAL GUIDELINE}

The epidemiology of Clostridioides difficile (CD) infection (CDI) has become more worrying in the past years, with a prevalence reaching $5.4 \%$ in a multinational European Centre for Disease Prevention and Control (ECDC) coordinated point prevalence survey, representing $48 \%$ of the gastrointestinal (GI) hospital care associated infections. The same study outlines an increased prevalence compared to the previous studies and also reports an increase in polymerase chain reaction (PCR) ribotype 027, associated with worse patient outcome [1].

Moreover, the EUCLID study reported the prevalence of ribotype 027 strains to be among the highest in Europe in Romania [2] and a recent study performed in tertiary centers from 6 cities in different areas from Romania identified a mean prevalence of 5.2 cases per 10,000 patient days, with the highest prevalence of PCR ribotype 027 of $82.6 \%$, values which are higher compared to the one previously reported [3]. Specific patients' category are at a higher risk for CDI, with a recent study performed in two tertiary centers in Romania highlighting a $27.6 \%$ rate of CDI in octogenarian patients [4]. Considering this epidemiological data, it is important to expand the therapeutic options, especially if we further take into account that $15.2 \%$ of patients experience recurrence within 8 weeks from the initial episode of CDI, while for patients with previous recurrences, the risk further increases up to $40.6 \%[5,6]$. Moreover, CDI is associated with high management costs, particularly in recurrent cases [7]. 
In this context, the use of fecal microbiota obtained from healthy individuals was shown to represent an effective treatment option for recurrent and refractory CDI. A recent meta-analysis evaluated the efficacy of fecal microbiota transplantation (FMT) for both recurrent and refractory CDI, highlighting the effectiveness of this treatment option for both these situations and suggested the superiority of the lower gastrointestinal delivery in obtaining good outcome. However, both routes of FMT delivery resulted in over $85 \%$ effectiveness [8]. In Romania, isolated cases successfully managed using FMT [9] and one prospective study comprising 30 patients with recurrent CDI [10] was reported. Moreover, a recent study performed in one tertiary center in Romania revealed some aspects regarding FMT perception among medical students that require improvement [11]; consequently, better knowledge on this procedure needs to be promoted among Romanian practitioners.

Considering that Romania does not have an available national guideline to offer standardization and foster the implementation of the method, this may lead to impairment of the efficacy and safety of this method and could also lead to medico legal issues. Despite strong evidence and recommendation of implementing FMT centers for CDI, following the 2017 European Consensus covering this issue [12], in many European countries there is no national guideline to support implementation of this therapeutic option.

Consequently, our aim was to create a national guideline for FMT in Romania, adapting the currently available guideline of the British Society of Gastroenterology and Healthcare Infection Society Guideline [13], the recent Consensus report of the UEG working group on stool banking [14], and following the recommendations of the European Consensus on FMT [12] in clinical practice, taking into account the local conditions and also the need to adapt FMT practice in the context of COVID-19 pandemic [15]. Moreover, following this initiative, stool banking can be consequently developed and the extension and adaptation of FMT for pediatric use in local conditions could also be established. Therefore, these would represent important steps towards improving healthcare in Romania, contributing to minimizing disparities between health care systems in Eastern Europe, compared to Western European countries. Hopefully, the implementation of FMT will lead to reducing mortality associated with CDI and also to reducing health care costs related to its management.

\section{DEFINITIONS}

Clostridioides difficile infection is defined as diarrheal stools or toxic megacolon and positive $\mathrm{CD}$ toxin $\mathrm{A}$ and/or B in stools or pseudomembranous colitis revealed by lower GI endoscopy; or colonic histopathology characteristic of CD infection (with or without diarrhea) on a specimen obtained during endoscopy, colectomy or autopsy [16].

Community-associated CDI is defined as the onset of CDI outside a healthcare facility or within 48 hours following admission to a healthcare facility without contact from a healthcare facility within the previous 12 weeks $[16,17]$.

Healthcare-associated CDI is defined as CDI with onset of symptoms: on day three or later, following admission to hospital or in the community within four weeks of discharge from a healthcare facility [17].

Clostridioides difficile colitis is characterized by several endoscopic findings such as bowel wall edema, erythema, friability, inflammation and pseudomembranes (raised yellowish or white plaques up to $2 \mathrm{~cm}$ in diameter over the colonic mucosa) which are highly suggestive for CDI [18].

Clostridioides difficile colonization is defined as the detection of the CD toxins in the absence of CDI symptoms [19].

Non-severe CDI is defined as at least 3 diarrhea stools (loose or watery) per day for 2 or more consecutive days, as the only symptom [19].

Severe CDI is characterized as an elevation in serum creatinine $>1.5$ times above the patient's normal levels, white blood cell count $\geq 15,000$ cells $/ \mu \mathrm{L}$, or serum albumin $<1.5 \mathrm{~g} / \mathrm{dL}$ within 2 weeks from symptom onset [20]. The severity of colitis can be guided following the presence of ongoing abdominal pain, fever $>38^{\circ} \mathrm{C}$ or white blood cell count $>15,000$ cells $/ \mu \mathrm{L}$ despite oral vancomycin at a dose of $500 \mathrm{mg}$ four times daily for at least 5 days [21].

Recurrent CDI (rCDI) is defined as diarrheal stools with a positive laboratory test after the end of treatment more than 2 weeks and less than 8 weeks following the onset of a previous episode $[13,16]$.

Refractory CDI is defined as lack of response to medication after 5 days or more of treatment [14].

Fecal microbiota transplantation is defined as the transfer of stool's biological material containing a community of microorganisms from a human donor to a human recipient with the aim of modifying the microbiota of the recipient [22].

\section{METHODOLOGY FOR GUIDELINE DEVELOPMENT}

The process of developing the national guideline aimed at shaping evidence-based recommendations for the use of FMT in clinical practice, adapting them to the current local conditions in Romania.

The guideline development process included the following steps: selection of the expert panel and members and calls for establishing the working group (WG), identification of main topics and generation of WG, development of statements according to the currently available evidence [12, 13, 23], review and adjustment of statements through online meetings and feedback after each session in order to release the final version of statements.

Original studies (prospective randomized, double-blinded, placebo-controlled studies) were included for the development of the guideline. In the absence of such studies, the evidence of lower quality was considered, including observational and retrospective studies or case series, as well as systematic reviews and meta-analyses. The data sources were found in the following electronic databases: PubMed, Scopus, ScienceDirect, Cochrane Library and Google Scholar, as well as recommendation statements and guidelines published on the websites of international scientific societies. The literature review and analysis were limited to articles published in either English or Romanian. 
A total number of 6 experts from 4 European countries constituted the experts' panel and 9 members constituted the WG from Romania.

The quality of evidence and strength of recommendation for each statement were determined according to the Grading of Recommendations Assessment, Development and Evaluation (GRADE) system [24]. Definition of the strength of recommendation and the quality of the published evidence are detailed in Supplementary file (Supplementary Tables I and II).

Initial statements were edited after the first voting session and discussed during a second online meeting. For each statement, consensus members were requested to rate their level of agreement, among the following: (1) agree strongly; (2) agree with minor reservation; (3) agree with major reservation; (4) disagree with major reservation; (5) disagree with minor reservation; (6) disagree strongly. If the rating was other than 'agree strongly' respondents were requested to add some comments to explain their reservation/disagreement, and how to improve the statement. For each statement, the pre-established threshold was reached when the overall result was $\geq 80 \%$, with the experts agreeing either strongly or with reservation. All statements not reaching $80 \%$ of agreement were revised and discussed during online meeting and following feedback.

The second version of the statements was sent to all members of the working group for the second round of feedback. The experts were allowed to adjust their answers in subsequent rounds. After the feedback was received, the statements were updated, and first manuscript draft was sent for revision of statements and comments.

Experts and all WG members provided commentaries and supporting literature for each statement. Finally, all members approved the final version of the released statements and commentaries.

\section{SUMMARY OF RECOMMENDATIONS}

\subsection{Which patients with CDI should be considered for FMT?}

\subsubsection{Indication for FMT}

4.1.1.1. Recurrent CDI. We recommend that FMT should be considered for patients with rCDI who have had at least two recurrences, or those who have had one recurrence and have risk factors for further episodes, including severe CDI (GRADE of evidence: high; strength of recommendation: strong).

4.1.1.2. Refractory $C D I$. We recommend that FMT could be considered in cases of refractory CDI (GRADE of evidence: low; strength of recommendation: strong).

4.1.1.3. FMT as initial therapy for CDI. We recommend that FMT should not be administered as an initial treatment for CDI (GRADE of evidence: low; strength of recommendation: strong).

4.1.1.4. Apart from CDI, there is insufficient evidence to routinely recommend FMT for any other GI or non-GI disease (GRADE of evidence: moderate; strength of recommendation: strong).
4.1.2. Place of FMT within therapeutic approach for CDI

i. We recommend consideration of treatment with extended/pulsed vancomycin and/or fidaxomicin before considering FMT as treatment for recurrent CDI in patients with severe or complicated CDI. (GRADE of evidence: low; strength of recommendation: strong).

ii. FMT could be considered in this setting when medication associated with a reduced risk of recurrence (e.g., fidaxomicin and bezlotoxumab) are not available. (GRADE of evidence: low; strength of recommendation: strong).

\subsubsection{Immunocompromised patient}

Indication for FMT in immunocompromised patients should be considered on a case-by-case basis and only after extensive donor screening. Although FMT seems safe, we recommend initial consideration of alternative CDI management for patients on major immunosuppressive agents including high-dose corticosteroids, calcineurin inhibitors, mammalian target of rapamycin (mTOR) inhibitors, lymphocyte-depleting biological agents, anti-tumor necrosis factor agents, and other chemotherapeutic antineoplastic agents in the last 12 weeks, uncontrolled HIV (CD4 count < 240 cells/mm 3 ), recent bone marrow transplant (within past 6 weeks) before offering FMT. (GRADE of evidence: moderate; strength of recommendation: weak)

\subsubsection{Contraindications}

Contraindications are represented by pregnancy, diagnosed with toxic megacolon / have anatomic abnormalities that could impact delivery method, risk for aspiration with naso-gastric (NG) tube, food allergy with previous anaphylaxis (GRADE of evidence: low; strength of recommendation: strong).

\subsection{Post-FMT follow-up, outcomes and adverse events}

\subsubsection{Definition of cure/remission}

We recommend that a decision regarding cure/remission for CDI should be recorded during follow-up. Clinical cure should be defined within 8 weeks, in the absence of diarrhea. Initial response to FMT should be defined on a case-by-case basis. (GRADE of evidence: very low; strength of recommendation: moderate)

\subsubsection{Definition of treatment failure post-FMT for CDI}

We recommend that treatment failure/recurrence should be defined on a case-by-case basis. Routine testing for CD toxin after FMT is not recommended, but it is appropriate to consider in the case of persistent CDI symptoms/suspected relapse. Recurrence of CDI should be defined as re-occurrence of CDI at more than 2 weeks and before 8 weeks after the onset of the initial CDI episode. (GRADE of evidence: low; strength of recommendation: strong).

\subsubsection{PostFMT immediate management}

Patients should be monitored for at least one night hospital stay, to document: clinical improvement, stool frequency reduction ( $<3$ stools in 24 hours), improvement of 
stool consistency ( $<5$ on the Bristol stool chart), absence of fever, improvement in vital signs - hypotension, tachycardia and/or signs of shock, improvement of other parameters of disease severity, laboratory - fall in white cell count and other inflammatory markers such as C-reactive protein (GRADE of evidence: very low: strength of recommendation: weak.

\subsubsection{Management of FMT failure}

Initial management of FMT failure could be by a further FMT infusion or anti-microbial therapies. The management of second FMT infusion failure should be established on a case-by-case basis. (GRADE of evidence: low; strength of recommendation: strong).

\subsubsection{General approach to follow-up post-FMT}

i. We recommend that patients should be warned about the short-term adverse events, in particular the possibility of self-limiting GI symptoms. They should be advised that serious adverse events are rare (GRADE of evidence: very low; strength of recommendation: strong).

ii. We recommend that all FMT recipients should routinely receive follow-up. Clinicians should follow-up FMT recipients for long enough to fully establish efficacy/adverse events, and for at least 8 weeks in total (GRADE of evidence: low; strength of recommendation: strong).

iii. At discharge, patients should be advised to be alert to symptoms of recurrent CDI in the first 4 weeks after FMT, even if the clinical response was obtained. We recommend that patient monitoring after initial favorable evolution after FMT should include a telephone call at 1 week after FMT counseling patients to be alert to symptoms and assessing primary non-response; telephone call or clinic assessment at week 4 to evaluate the patient for a secondary non-response; clinical assessment at 8 weeks; telephone call at 12, 24 weeks (GRADE of evidence: low; strength of recommendation: weak).

\subsection{What recipient factors influence the outcome of FMT} when treating people with CDI?

\subsubsection{Special patient categories}

i. We suggest that FMT should be offered with caution to several patients' categories, including CDI and decompensated chronic liver disease, solid organ transplant (SOT), intensive care unit (ICU) patients (GRADE of evidence: very low; strength of recommendation: weak).

ii. We suggest that FMT could be offered with caution to patients with CDI and decompensated chronic liver disease, after case-to-case judgment and risk stratification, including CHILD and Model for End-Stage Liver Disease (MELD) scores, albumin level. (GRADE of evidence: very low; strength of recommendation: moderate.

iii. FMT can be considered in solid organ transplantation recipients; prior use of other available therapies, including tapered Vancomycin regimen or Fidaxomycin is recommended in this setting. (GRADE of evidence: low; strength of recommendation: low)

iv. FMT appears to be effective in selected patients, who develop severe and/or recurrent CDI and require ICU, but best directed by involving all stakeholders relevant to patient's care. The multidisciplinary team should preferably include a ICU therapist, an infectious disease specialist, a gastroenterologist and surgeon, and consider the route, timing of FMT and potential alternative options. In the ICU setting, NG tubes already in place might represent the preferable route of administration. (GRADE of evidence: low; strength of recommendation: strong)

\subsubsection{Other comorbidities and FMT}

We recommend that FMT should be considered for appropriate patients with recurrent CDI regardless of other comorbidities, exerting caution when several characteristics for potential increased risk of early FMT failure are present, such as: use of non-CDI antibiotics within 8 weeks of FMT, history of CDI-related hospitalization, severe or severecomplicated CDI, pseudomembranous colitis, low serum albumin concentration. (GRADE of evidence: low; strength of recommendation: strong)

4.4. What donor factors influence the outcome of FMT when treating people with CDI?

\subsubsection{General approach to donor selection}

Where possible, FMT is best sourced from a centralized stool bank, from a healthy unrelated donor (GRADE of evidence: low; strength of recommendation: weak).

\subsubsection{Age and body mass index restrictions for potentia} donors

We suggest that people should only be considered as potential FMT donors if they are $\geq 18$ and $\leq 60$-years-old and have a body mass index (BMI) of $\geq 18$ and $\leq 25 \mathrm{~kg} / \mathrm{m} 2$ (GRADE of evidence: low; strength of recommendation: weak).

\subsubsection{General approach to the donor screening} assessment

i. It is mandatory to screen potential donors by questionnaire and personal interview, to establish risk factors for transmissible diseases and factors influencing the gut microbiota (GRADE of evidence: low; strength of recommendation: strong).

ii. Biochemical evaluation for the screening of the donor is compulsory and should include the following: pathogen screening (Hepatitis A IgM, Hepatitis B (HBsAg and HBcAb), Hepatitis C antibody, Hepatitis E total antibodies*, HIV-1 and HIV-2 antibodies, HTLV-1 and HTLV-2 antibodies*, Treponema pallidum antibodies (TPHA, VDRL), EpsteinBarr virus and IgG and IgM, Cytomegalovirus IgG and IgM), general metabolic screening (Full blood count, Creatinine and electrolytes, Liver enzymes (including albumin, bilirubin, ALAT, ASAT, GGT, ALP), C reactive protein) fecal sample testing (CD PCR, Campylobacter PCR, Salmonella, and Shigella by standard stool culture, Shiga toxin-producing Escherichia coli by PCR, Multi-drug resistant bacteria: CPE, ESBL, VRE, MRSA, Stool ova, cysts and parasite analysis, including for Microsporidia; Acid fast stain for Cyclospora and Isospora, fecal antigen for Cryptosporidium and Giardia, Helicobacter pylori fecal antigen, Norovirus, rotavirus Antigen ${ }^{\star}$ ), PCR SARS CoV2 nasopharyngeal swabs at $48 \mathrm{~h}$ 
interval and stool PCR (* - will require evaluation only when FMT will be addressed to immunosuppressed recipients).

4.4.4. Repeat donor checks, and donation pathway

We recommend that a repeat health questionnaire should be assessed at the time of each stool donation. To ensure ongoing suitability for inclusion as a donor, laboratory screening should also be repeated regularly at 3 months (GRADE of evidence: low; strength of recommendation: weak).

4.5. What factors related to the preparation and administration of the transplant influence the outcome of FMT when treating people with CDI?

\subsubsection{General principles of FMT preparation}

i. We recommend that stool collection should follow a standard protocol and that donor stool should be processed within 6 hours of defecation (GRADE of evidence: low; strength of recommendation: strong).

ii. We recommend that sterile $0.9 \%$ saline should be considered as an appropriate diluent for FMT production, and cryoprotectant such as 10-15\% glycerol or maltodextrintrehalose cocktail recipes in the ratios of $3: 1 /$ should be added for frozen FMT, stored in a $-80^{\circ} \mathrm{C}$ standard freezer, and then later thawed at room temperature). (GRADE of evidence: moderate: strength of recommendation: strong)

iii. We recommend using at least $50 \mathrm{~g}$ of stool in each FMT preparation (GRADE of evidence: moderate: strength of recommendation: strong).

iv. We suggest that homogenization and filtration of FMT should be undertaken in a closed disposable system (GRADE of evidence: low; strength of recommendation: weak).

\subsubsection{Fresh versus frozen FMT}

We recommend that only the use of banked frozen FMT material should be considered. The use of fresh preparations should be avoided due to safety concerns. (GRADE of evidence: high; strength of recommendation: strong).

\subsubsection{Use of frozen FMT}

i. We recommend that FMT material stored frozen at $-80^{\circ} \mathrm{C}$ should be regarded as having a maximum shelf life of 12 months. Refreezing once defrosted is not permitted. (GRADE of evidence: low; strength of recommendation: strong).

ii. We suggest consideration of thawing frozen FMT at an ambient temperature and using within 6 hours of thawing (GRADE of evidence: low; strength of recommendation: weak).

iii.. We suggest not thawing FMT in warm water baths, due to the risks of cross contamination with Pseudomonas (and other contaminants) and reduced bacterial viability (GRADE of evidence: very low; strength of recommendation: weak).

\subsubsection{Use of specific medications in the period around} FMT administration

\subsubsection{Diet and bowel lavage}

i. We recommended a clear liquid diet one day prior FMT and bowel lavage to be administered prior to (the evening before) FMT via the lower GI route in the absence of contraindications; polyethylene glycol preparation is preferred.
(GRADE of evidence: low; strength of recommendation: strong)

ii. Bowel lavage could also be considered prior to FMT via the upper GI route in the absence of contraindications (for patients with renal impairment adjustment of PEG quantity, bowel cleansing should be avoided in patients with severe CD colitis and in patients with IBD and CDI, where low-volume PEG preparations are preferred. (GRADE of evidence: low; strength of recommendation: low).

4.5.4.2. We recommend that proton pump inhibitors (PPIs) use should be avoided periprocedurally, considering conflicting results on PPIs' effect in this setting. (GRADE of evidence: moderate; strength of recommendation: low)

4.5.4.3. We suggest the use of a single dose loperamide (4 mg) on the morning of the procedure, preferably at least 2 hours before procedure, when FMT is delivered via lower GI and in the absence of severe colitis. (GRADE of evidence: low; strength of recommendation: weak).

4.5.4.4. Additional antibiotics pre-FMT. We recommend the administration of further antimicrobial treatment for CDI for at least 72 hours prior to FMT; the preferred antibiotics should be either vancomycin or fidaxomycin. (GRADE of evidence: low; strength of recommendation: strong).

4.5.4.5. Washout period between antibiotic use and FMT

i. To minimise any deleterious effect of antimicrobials on the FMT material, we recommend that there should be a minimum washout period of 24 hours between the last dose of antibiotic and treatment with FMT (GRADE of evidence: low; strength of recommendation: strong).

ii. We suggest considering consultation with infectious disease specialists or medical microbiologists for advice whenever FMT recipients also have an indication for long term antibiotics or have an indication for non-CDI antibiotics within 8 weeks of FMT (GRADE of evidence: very low; strength of recommendation: weak).

\subsubsection{Route of FMT delivery}

i. We recommend that colonoscopic administration of FMT as a first choice route for the treatment for recurrent or refractory CDI should be used where appropriate/applicable. Other preferred options include duodenal delivery via endoscopy; enema; only when these options are not applicable, a nasogastric tube can be used. (GRADE of evidence: high; strength of recommendation: low).

ii. Where colonoscopic administration is used, we suggest considering preferential delivery to the caecum or terminal ileum, as this appears to give the highest efficacy rate (GRADE of evidence: low; strength of recommendation: weak).

iii. Delivering FMT via an ileostomy as treatment of CDI appears to be safe and effective in the treatment of rCDI. (GRADE of evidence: low; strength of recommendation: strong)

\subsubsection{Number of infusions}

At least 2 infusions should be considered in patients with rCDI showing signs of non-response (persistent diarrhea at $48 \mathrm{~h}$ from first infusion) (GRADE of evidence: moderate; strength of recommendation: strong). 
4.5.7. We recommend that best practice for prevention of further transmission of CDI should be applied throughout when administering FMT to patients with CDI (nursing with enteric precautions, sporicidal treatment of endoscope, etc) (GRADE of evidence: high; strength of recommendation: strong).

\subsection{Basic requirements for implementing a FMT service}

\subsubsection{General considerations}

In Romania, FMT will be performed under the regulations of the Ministry of Health, only in credentialed university centers, where infrastructure is available. (GRADE of evidence: low; strength of recommendation: strong).

4.6.2. Minimum infrastructure requirements should be fulfilled in order to achieve microbiological safety (outpatient room, sample FMT preparation and storage rooms with separate ventilation system and controlled environmenttemperature, humidity). Minimum equipment include category 2 hood, clean but not sterile, equipped with scale, stomacher, separate ventilation system. (GRADE of evidence: moderate; strength of recommendation: strong)

\subsubsection{FMT manufacturing and production quality} control

i. We recommend ensuring the traceability of supply (GRADE of evidence: very low; strength of recommendation: strong).

ii. We recommend monitoring, notification and investigation of all adverse events and reactions related to FMT (GRADE of evidence: very low; strength of recommendation: strong).

iii. We recommend ensuring the traceability of supply, using a quality system that relies on the principles of good clinical practice. (Quality of evidence: law governed; grade of recommendation: strong

iv. We recommend the creation of at least a local database (preferably a national database) including FMT recipients' data with careful monitoring of response to FMT and report adverse events, for a minimum 8 week follow-up, and a donor registry minimally including the date when donor screening process had begun and was completed, the date of the last completed screening process. (GRADE of evidence: low; strength of recommendation: strong).

\subsubsection{Donor screening governance}

We recommend each center to establish a local clinical governance board, including at least a gastroenterologist, infectious disease specialist, microbiologist and epidemiologist.

(GRADE of evidence: low; strength of recommendation: strong)

\subsubsection{Credentialing and recredentialing of FMT centers}

i. Credentialing should include the evaluation of the performance indicators of the activity of the center.

ii. Interval for recredentialing should be 3 years iii. The credentialing and recredentialing process must include: National Insurance House, the Ministry of Public Health, the Public Health Regulatory Council (Directia de Sănătate Publică județeană) and professional Societies: Romanian Society of Gastroenterology, Romanian Society of Microbiology.

\subsubsection{Multidisciplinary teams}

We recommend that a multidisciplinary team should be formed to deliver FMT services (GRADE of evidence: very low; strength of recommendation: strong).

\section{RATIONALE FOR RECOMMENDATIONS}

\subsection{Which patients with CDI should be considered for} FMT?

There is significant evidence for the efficacy and safety of FMT for the treatment of recurrent CDI [25-30]. In defining recurrent CDI, there have been various definitions, mainly relying on a minimum threshold of the return of clinical symptoms (e.g., at least three unformed bowel movements within 24 hours, for at least 2 consecutive days), with the latest ECDC definition including the reappearance of symptoms in the following 2 to 8 weeks after previous successful CDI treatment $[1,28,31]$. Moreover, the current data are relatively heterogeneous concerning the methodology for performing FMT and also the definition used for the resolution of CDI [8].

The choice of FMT in this setting should balance the risks and benefits and should guide a joint decision of either FMT or (tapered) antibiotics with or without additional treatment with e.g. monoclonal antibody or nonabsorbable antibiotics. One randomized controlled trial (RCT) performed by Hvas CL et al. [30] showed that FMT after vancomycin administration was superior to fidaxomicin and vancomycin monotherapies for rCDI for combined clinical and microbiological resolution and clinical resolution alone.

For refractory CDI, even though exclusion of other causes is required (for example ulcerative colitis, microscopic colitis), FMT as rescue treatment appears encouraging, although few studies have addressed the effects of FMT in patients with refractory or severe CDI [32-37]. Furthermore, FMT appeared to decrease mortality in patients with severe CDI refractory to antibiotic therapy [34-36, 38], and could also be considered as a therapeutic option for this condition, with decision taken on a case-by-case basis.

Considering some particular patient-related aspects of CDI infection in potential FMT recipients in Romania, such as increased age [4] and also the prevalence of ribotype 027 [3], the working group discussed the opportunity for the use of FMT among patients with one CDI recurrence and risk factors for other recurrences. We considered the risk factors identified in literature, namely antibiotics use for non-C. difficile after CDI diagnosis, long term gastric acid suppression, severe underlying disease and/or renal insufficiency/ severe illness by Horn index, previous severe CDI, prolonged hospital stays, severe underlying disease and/or renal insufficiency, a history 
of previous CDI, previous CDI severity, prolonged hospital stay hypervirulent strain, NAP1/BI/027 $[39,40]$. Although there is limited evidence in RCTs, experts agreed that FMT could be indicated after one recurrence for those with features of severe disease or risk factors for recurrence.

Nowadays there is increasing interest in exploring wider opportunities for FMT in the course of various disease although studies confirming the efficacy of FMT in this setting are limited or contradicting. Among areas with promising results is the use of FMT for ulcerative colitis (UC), with significant difference in achieving remission reported in two RCTs including UC patients undergoing colonoscopically administered FMT, with prior bowel lavage $[41,42]$. However, another RCT including UC patients who were administered FMT via duodenal tube did not identify significant differences in achieving remission [43]. One RCT including Crohn's disease patients with colonic or ileocolonic disease, who were administered FMT colonoscopically, with prior bowel lavage identified no difference in sustained engraftment between allogeneic FMT and sham, but a decrease in Disease Endoscopic Index of Severity at 6 weeks was obtained in the group receiving FMT [44]. Regarding the use of FMT for irritable bowel syndrome, among the 7 RCTs available to this moment, all with various study protocols and routes of administration, conflicting results have been reported [45-50].

Extra-digestive indications which are studied for FMT include hepatic encephalopathy, with an RCT showing significant reduction in new hepatic encephalopathy episodes among cirrhotic patients receiving FMT [51] and potential for eradication of multidrug resistant organisms, with a randomized open label study which indicating a lightly decreased ESBL-E/CPE carriage among patients who received FMT [52]. Taking into account all the above-mentioned evidence, FMT for other indications than CDI should preferably be currently limited only to the research setting or, in the absence of alternative therapeutic option.

\subsubsection{Place of FMT within therapeutic approach for CDI}

At least two licensed agents (fidaxomicin and bezlotoxumab), are available having demonstrated they significantly reduce the risk of CDI recurrence compared with vancomycin. [53, 54]. These potential medications should also be considered for recurrent CDI.

However, considering evidence suggesting the superiority of FMT over the use of fidaxomicin in recurrent FMT (30) and also the financial limitations of using fidaxomicin or bezlotoxumab in Romania, the local members of the working group strongly encouraged using FMT for rCDI after failure of extended/pulsed vancomycin. Moreover, since it has been suggested that additional antibiotic treatment with vancomycin after first FMT infusion, followed by a second FMT, could contribute to improving outcomes in patients with severe CDI $[55,56]$. However, in most case reports, the patients responded to a single FMT without additional antibiotic treatment, suggesting that a combined treatment is not necessary in all patients. More research is needed to determine the additional value of sequential FMTs followed by antibiotic treatment, especially in relation to the presence or absence of pseudomembranous colitis.

\subsubsection{Immunocompromised patient}

Although there is increasing evidence that FMT in immunocompromised patients appears safe [57], there are still reports of severe adverse events, including death, following transfer of multi-drug resistant organism calls for particular caution in this patient group [58]. Food and Drug Administration (FDA) issued a safety alert On June 13,2019 , regarding the risk of serious adverse reactions due to transmission of multi-drug resistant organisms (MDRO) through FMT to two immunocompromised patients, among which one resulted in death [59]. In this particular case, the donor had not been screened for MDRO. Consequently, the inclusion of MDRO screening in all active and future FMTbased study protocols must be performed and the precise status of immunocompromised patients will be determined by the members of the multidisciplinary team involved in FMT.

Since only limited data is currently available on the risks of FMT in patients at specific different levels of immunosuppression, the local members of the working group expressed the need for careful consideration when using FMT in immunocompromised patients and highly encourage judicious application of FMT in this patient category, along with thorough surveillance for and documentation of adverse events and long-term follow-up in immunocompromised patients.

\subsubsection{Contraindications for FMT}

There are no known absolute contraindications for FMT based on the current clinical evidence [60]. However, the procedure has not been adequately studied in pregnancy and should be delayed until the patient is postpartum [61].

Colonoscopy route of delivery is more effective than enema, although, when toxic megacolon is diagnosed or the patient has anatomic abnormalities the colonoscopy is contraindicated and the nasoduodenal route should be preferred [62].

Although no cases of allergy, anaphylaxis or anaphylactic shocks have been reported in the literature, the food allergies should be evaluated in recipients before FMT. If the patient reports a severe food allergy, either the patient should be evaluated by an allergist to confirm the allergy or, if the allergy is confirmed, the stool source should from a donor who has abstained from the allergenic agent for a period of 7 days [12]

\subsection{Post-FMT follow-up, outcomes and adverse events}

\subsubsection{Definition of cure/remission}

There are significant reports that symptoms of CDI resolve relatively fast after a successful FMT, although the timing for defining a favorable course varied between few hours in some studies [63] to an average of $4-5$ days in others [64].There is no uniformly agreed definition for treatment success post-FMT for CDI, but most evidence lays on clinical criteria, assessing treatment response as present when stool frequency decreases to less than 3 per 24 hours (or returning to the prior defecation frequency) over a period of at least 72 hours, criteria which can also be combined with improvement of disease severity parameters (clinical, biological).

Moreover, since ECDC definition of recurrence is between 2 weeks following treatment and before $8(1)$, the absence of 
symptoms within 8 weeks from the FMT is also to be considered when evaluating cure/remission [13]. The use of FMT as treatment for recurrent or refractory CDI infection and other potential indications: joint British Society of Gastroenterology and Healthcare Infection Society guidelines [13, 65]. The working group agreed that the definition should be made on a case-by-case basis but should include an assessment for cure/ remission of CDI within 8weeks.

\subsubsection{Definition of treatment failure post-FMT for CDI}

FMT failure can be defined only based on clinical criteria, as persistence of diarrhea after the procedure or recurrence of diarrhea after initial improvement within 8 weeks after the procedure [66] or using both clinical and laboratory testing for CDI, as diarrheal symptoms ( $\geq 3$ loose stools within a 24hour period) and a positive CD stool test. The WG agreed that definition on a case-by-case basis is adequate in this setting and that decision regarding cure/remission from CDI should be recorded during follow-up.

Considering that up to $80 \%$ of failures happen before week 4, with the mean failure time at 2 weeks after FMT [66], it is important to prior acknowledge potential risk factors for failure. These factors include: ongoing antibiotic use, age, number of admissions prior to FMT [32], antimicrobial exposure pre-FMT or continuing antibiotic use during or directly following FMT [67] and also severe CDI and inadequate bowel preparation [68]. However, it must be considered that a substantial number of recurrences occur after 8 weeks from FMT delivery and the appearance of a new CDI episode later than 8 weeks post-FMT should be regarded as a late recurrence [69].

\subsubsection{PostFMT immediate management}

There is often a relatively short period of post-procedural observation in patients receiving FMT $[27,31]$. Since, usually, most of the immediate side-effects of post-endoscopic administration of FMT are minor and self-limiting [70], most FMT centers usually manage patients by the standard protocols for an endoscopic procedure [71, 72], without any specific adaptations, apart from advising about the possibility of self-limiting GI side effects and following the protocol of departmental infection control. Consequently, the postprocedural period of observation is relatively short [21, 27], allowing patients to leave the administration site after the period of observation. However, overnight observation protocol was applied for a cohort of elderly patients with multiple comorbidities undergoing FMT [73].

Depending on the severity of CDI, patients should be monitored in order to evaluate initial treatment response, at least on clinical grounds, following several parameters such as stool frequency reduction ( $<3$ stools in 24 hours), improvement of stool consistency ( $<5$ on the Bristol stool chart), absence of fever, improvement in vital signs - hypotension, tachycardia and/or signs of shock [12]. Moreover, when baseline biochemical parameters have been modified, then at least the decrease in white cell count and other inflammatory markers such as C-reactive protein, fibrinogen should also be monitored.
Although in cases with non-severe CDI there is no need for an overnight hospital stay, the local members of the working group considered at least one night hospital stay would be useful for monitoring. This statement has been locally supported, considering that most cases of rCDI in Romania are often met in frail patients with comorbidities, exhibiting moderate-severe colitis and because access to prompt readmission in case of unfavorable evolution may be limited in some situations.

\subsubsection{Management of FMT failure}

FMT has been successful in controlled trials, but there is potential for treatment failure or incomplete resolution of patient symptoms. Patient expectations should be calibrated appropriately during the discussion. Predictors of treatment failure include severe and severe-complicated CDI, inpatient status during FMT, and the number of previous CDI-related hospitalizations [32] as well as underlying inflammatory bowel disease [74].

In a systematic review about FMT use for the treatment of CDI, a total of 25 case series and two RCTs were identified providing evidence on the efficacy of FMT for recurrent, refractory, or even an initial episode of CDI, with most studies targeting recurrent CDI [25]. The largest of these studies was an unblended RCT which compared FMT to oral vancomycin (14 days) or oral vancomycin (14 days) plus gastrointestinal lavage [27]. The study was terminated early due to a significant difference in recurrence between the FMT group and the two control groups. Apparently, $81 \%$ of patients in the FMT arm had sustained resolution of diarrhea after the first fecal transplant compared with $31 \%$ of patients in the vancomycin arm and $23 \%$ of those in the vancomycin plus bowel lavage arm. We should keep in mind that most of the study enrollees had four episodes of CDI before enrolment, making them a different population than other trials [27]. Furthermore, repeating FMT in patients who failed the first FMT found to improve the response rate to $94 \%[27,75]$. Another RCT of 39 adults with recurrent CDI demonstrated comparable efficacy of FMT (with median of three recurrences per patient) [25].

A two-week standard course of oral vancomycin or repeated instillation of feces collected from new donors might be a solution for patients who initially fail to respond to fecal instillation [68] and vancomycin taper associated with a lesser chance ( $85 \%$ reduction in risk) of a relapse following FMT [32]. A second administration of FMT by enema after a first failure (elderly patients possibly attenuated the sphincter tone in elderly patients) [76].

\subsubsection{General approach to follow-up post-FMT}

Most frequently reported short-term adverse events postFMT for CDI are mild, and include mainly self-limiting GI symptoms. These may include nausea $[21,27,60,72]$, bloating [25, 26, 30], belching [27], abdominal cramps/pain [26, 28, $29,31]$, diarrhea $[21,27,31]$. These symptoms typically resolve within a few hours to days $[21,27,72]$. Except for the short-term minor adverse events, long-term adverse reaction (such as postinfectious irritable bowel syndrome) [77] and also severe adverse events should be reported and a clear 
distinction between procedure-related versus unrelated need to be made.

Serious events related to the way of administering FMT are rare, but they occur and may be due to complications of endoscopy, such as perforation and bleeding or adverse effects related to sedation, such as aspiration (one death reported after aspiration during sedation for FMT administered via colonoscopy [60]; death of two patients from pneumonia on aspiration of feces administered to the duodenum via enteroscopy or nasoduodenal tube was reported [38,77].

Moreover, deaths after an initial positive response to FMT but with subsequent development of ileus and colonic perforation and death in individuals with the ribotype 027 CDI strain who never responded to FMT and died within three months [8] were also reported. There has also been reported transmission of enteric pathogens via FMT: cases of documented norovirus infection 2 days and 12 days after FMT [78], a case report of fever and Escherichia coli bacteremia 24 hours after FMT via colonoscopy in a patient with IBD [58] and infections caused by enteropathogenic Escherichia coli and Shiga toxin-producing Escherichia coli [79].

All side effects and severe side-effects should be reported and registered within the available local database by the treating physician, within 48 hours after occurrence, even if apparently initially not relatable to FMT, especially considering that usually they have been underreported $[77,80]$. In case of severe adverse events, prompt and effective procedures for the disposal of the used FMT material should be applied.

Follow-up of patients undergoing FMT is compulsory, but there is significant variability in the way which post-FMT follow-up was performed, both in terms of duration and modality (outpatient visits, telephone interviews, electronic diary or standardized questionnaires) $[66,81,82]$.

In the context of COVID-19, patients should be instructed to immediately contact the center where FMT was performed if symptoms of infection develop or if they are diagnosed with COVID-19 within 28 days of the procedure. Moreover, follow-up appointments at different time points (between 1 and 8 weeks) should be conducted as much as possible, via internet if possible, or via phone calls, in order to assess signs of recurrence and identify potential symptoms of infection, excepting the situations where direct assessment is mandatory [83].

After the most recent definition of rCDI from ECDS, most studies and current guidelines use a follow-up period of at least 8 weeks. Our WG decided that at least 8 weeks of follow-up was appropriate post-FMT to completely evaluate efficacy and potential adverse events. However, since there is some concern about long-term side effects of FMT, including weight gain, the development of IBD, cancer, autoimmune diseases, allergies, or neurological diseases, a national or at least local registries should be developed in order to perform long-term follow-up [84].

\subsection{What recipient factors influence the outcome of FMT} when treating people with CDI?

Special patient categories and other comorbidities: several patient characteristics such as immunocompromised state, decompensated liver cirrhosis, critically ill patients requiring intensive care unit (ICU), all of them with their own risks of unfavorable outcome. Although evidence has been limited for most groups, currently there is increasing data in immunocompromised patients, supporting the use of FMT for treatment of CD infection in immunocompromised patients, with similar rates of serious adverse events to immunocompetent patients [85]. FMT in immunocompromised patients appears to have comparable efficacy and safety data to those on patients with intact immunity, but practitioners should perform FMT in solid organ transplantation (SOT) recipients with refractory CDI with vigilance for infectious complications [86-88].

For SOT patients, there is data from a RCT suggesting FMT is safe. The study reported FMT-related adverse events in $22.3 \%$ of cases, mainly comprising self-limiting conditions including nausea, abdominal pain, and FMT-related diarrhea. Severe adverse events were registered in $3.2 \%$ of cases, with no FMT-related bacteremia; however, among cytomegalovirusseropositive patients' reactivation was reported. Repeated FMT or additional antibiotics may be needed to optimize rates of cure with FMT within this patient category [89] and consequently an indication for FMT should me more carefully considered in this patient category.

A study including cirrhotic patients, aiming to assess safety, tolerability and impact on microbiota and brain function in hepatic encephalopathy of capsulated FMT, demonstrated that oral FMT capsules appear to be safe, and even improved EncephalApp performance [90]. Another study including patients with chronic liver disease, among which 9 with cirrhosis and 1 recipient of liver transplantation, FMT was well tolerated, without infectious complications [91].

For critically ill patients receiving rescue FMT the procedure appears to be safe [92]. Moreover, in another study FMT provided mortality benefit over standard of care for severe and fulminant CDI and should be considered in critically ill patients [93]. The route of administration should be decided within the multidisciplinary team [94].

5.4. What donor factors influence the outcome of FMT when treating people with CDI?

\subsubsection{General approach to donor selection}

The selection of possible donors is one of the key points for FMT safety and efficacy. In principle, donors genetically related to FMT recipients showed similarities in their intestinal microbiota [94], because donors and recipients living in the same house are largely exposed to the same infectious risk factors and allergens [95]. However, FMT has also been successfully performed with donor feces from unrelated healthy volunteers [96]. The success rates of FMT for recurrent CDI are comparable for related and unrelated donors $(89.5 \%$ vs. 90.7\%) [97]. The advantage of donor feces in terms of relationship status for indications other than CDI remains uncertain. There is also a theoretical advantage of a common genetic background and shared environment, leading to better immunological tolerance of the FMT. Nevertheless, additional clinical evidence has proven no association between donor and FMT outcomes $[98,99]$, and either related or unrelated donors are acceptable in different countries $[13,100]$. Unrelated FMT volunteer donors may be more beneficial in cases where 
genetics could influence the disease outcome. Consequently, no specific recommendation is made, and the donor can be a family member or have no relationship with the recipient. In all cases, the same screening tests should be carried out.

\subsubsection{Age and body mass index restrictions for potential} donors

Although there is currently no consensus on age restrictions for potential FMT donors, many studies included donors between 18 and 60 years old, potentially due to the fact that there is a decrease in gut microbiota stability and diversity in people who are 60 years and older $[101,102]$ and also that there might be undiagnosed oncologic or cardiovascular comorbidity in potential donors over 60 years of age.

As far as BMI restrictions are concerned, there have been case reports of weight gain in a recipient following FMT from an overweight donor [103]. On the contrary, a large retrospective cohort study highlighted that single FMT did not determine weight gain in FMT recipients [104]. As it is currently recommended in the available guidelines, donors exciding the normal BMI range of 18-25 should be excluded from becoming stool donors, until further prospective studies confirm otherwise $[12,13]$.

\subsubsection{General approach to the donor screening} assessment

An extensive screening questionnaire and a strict panel of tests for donor screening are recommended prior to FMT in order to reduce and prevent the occurrence of adverse events. Both the guidelines in the United States and European consensus conference suggest to use a donor questionnaire to evaluate the exclusion and inclusion criteria. The donors should undergo an additional interview on the same day of the donation. Standard donor screening protocols should be set up to decrease the risks of infection transmission from the donor to recipient, and it should include both blood and stool examinations within 4 weeks before donation (Supplementary Table III)

The incidence of SARS-CoV-2 infection raised concerns about the potential for transmission through feces, and FDA released a safety alert on FMT and donor screening in March 2020 [105]. The safety alert recommended that a donor stool obtained after December 1, 2019 should be tested for SARSCoV-2 before being used to treat patients. Subsequently, an international expert panel proposed donor screening measures including PCR assays of nasopharyngeal swab samples and direct testing of donor feces once a test is available, given the uncertainties around the risk of transmission via FMT [15, 83], although there are no data on the clinical outcomes in patients who received a donor stool from an infected donor with SARS-CoV-2.

In addition, screening for transmissible diseases or factors disturbing the gut microbiota should be conducted with a questionnaire and personal interviews $[13,100]$ and comprehensive blood and stool tests are both prerequisites [100].

If the donor is considered a qualified candidate, the stool donation should be conducted within 4 weeks [12]. An interview on the day of the donation should be conducted to check for potentially health issues with recently onset [12]. The donor's stool should be processed within $6 \mathrm{~h}$ of defecation [13]. Besides, the donor is asked to be interviewed again 1-2 months after the stool donation. If there are any suspicious risk factors noted during follow-up, further examinations and surveillance are mandatory [13], along with quarantine of the sampled stool for 2 months in view of safety regarding SARSCoV-2 transmission. If the eligible donor is willing to donate stool again, the screening process shall be conducted at least every 4 months [13].

\subsubsection{Repeat donor checks}

Donors repeatedly used for FMT should be retested every 3 months, and sooner in the case of certain risks for infectious diseases (e.g. vacation in countries with a high risk for gastrointestinal infections, contact or history of COVID-19).

5.5. What factors related to the preparation and administration of the transplant influence the outcome of FMT when treating people with CDI?

\subsubsection{General principles of FMT preparation}

The stools are collected in a disposable container with a wide opening and a tight lid, and the sample for FMT must not contain urine. Preparation of stool is carried out in a dedicated room. It is performed in a biosafety cabinet to avoid the risk of cross-contamination and ensure the protection of personnel. All preparation steps are performed at room temperature.

Preparation of fecal material from donor is as follows: $200 \mathrm{~mL}$ of diluent (water or $\mathrm{NaCl} 0.9 \%$ ) was added to $50 \mathrm{~g}$ of stool and homogenized into a liquid suspension, placed in the bag mixer. Reports have shown that FMT is more effective if amount of feces used is more than $50 \mathrm{~g}$ increases, in comparison to the use of feces more than $50 \mathrm{~g}$ ) [106] (Supplementary file, Table IV). Next, the mixture was allowed to settle for $5 \mathrm{~min}$ and then filtered first through the gauze. Filtrate obtained in the syringes can be frozen and thawed without loss of efficiency, which allows long-term storage of those materials. Also, the fecal materials obtained from the donor can be frozen at $-80^{\circ} \mathrm{C}$ with the addition of glycerine [107]. Glycerol is added to the liquid preparation (final concentration, 10\%) for storage, to increase the viability of the microbiota [71].

It is essential to ensure rigorous traceability of the FMT. For this purpose, biological samples from the donor should be stored and a batch file must be created and archived. For each FMT, two samples from the donor are stored at $-80^{\circ} \mathrm{C}$. Stool collection: $1-2 \mathrm{~g}$ of stool collected at the time of the donation, in a polypropylene tube Sample collection: $1-5 \mathrm{~mL}$ of preparation administered to the recipient in a polypropylene tube

\subsubsection{Fresh versus frozen FMT}

Recent severe adverse events due to inadvertent transfer of pathogens during FMT in the US and the potential for transmission of SARS-CoV-2 during FMT make it imperative that all fecal samples used for FMT are thoroughly screened. This requires a period of quarantine between stool processing and use of FMT which is not possible using fresh stool. We therefore do not recommend the use of fresh stool samples for FMT. In summary, the use of frozen product is logistically less 
cumbersome, and it is also advisable from the point of view of patient safety, especially in the current context of COVID-19 pandemic.

\subsubsection{Frozen FMT}

The simplest way to process the donor's microbiome is to liquefy the donor stool in normal saline and then filter it to eliminate the cellular component. This suspension will be frozen at -20 to $-80^{\circ} \mathrm{C}$ for later use. The main advantage of the frozen product is its ready availability whenever needed, excluding a time-consuming donor screen and preparing the stool sample for administration on short notice. In this case the composition of the microbiota and their viability will then remain unchanged, even after six months of storage at -20 to $-80^{\circ} \mathrm{C}[25,26]$. Successful treatment of rCDI with samples that have been stored for 9 to 12 months has been reported. Multiple retrospective analyses and randomized trials [21, 71, $108,109]$ have compared the efficacy of fresh and frozen FMT without revealing any significant difference.

\subsubsection{Use of specific medications in the period around} FMT administration

\subsubsection{Diet and bowel lavage}

Bowel lavage has been used prior to FMT in order to remove residual antibiotics which could impair the engraftment of transplanted microorganisms, and also to remove residual CD spores and vegetative cells [110112]. It has already been stated that the efficacy of FMT may depend upon the technique used to cleanse the colon before administration of the FMT [113] Bowel lavage with polyethylene glycol preparations is usually recommended with very good results, especially before FMT administration via colonoscopy [31], but it is sometimes also prescribed with good results before FMT administration via upper GI route $[27,38,114]$. It is not known if bowel lavage is required before donor feces infusion but given the excellent results of FMT after bowel lavage using polyethylene glycol preparations, it is generally prescribed. However, FMT can be considered without bowel lavage as well, as it has been done in several studies, apparently without a reduction in efficacy [21].

Positive results have also been reported for subjects who stopped antibiotics or probiotics 48 hours before the procedure, followed a clear liquid diet a day before FMT, and received 10 oz. of magnesium citrate or Golyetly for subjects with kidney function impairment the night before the procedure [115]. Since IBD patients represent a special patient category when discussing bowel preparation, it is important to consider lowvolume preparations for these patients, since they are well tolerated, without more frequent side effects in patients with relatively inactive IBD than the general population, as it has been emphasized by a meta-analysis focusing on cleansing preparations in patients with IBD [116]. It is still unclear whether this could be safely administered to patients with more active IBD and CDI, or whether tap water enema could be an alternative. For the moment, the working group concluded that caution when recommending bowel lavage in IBD patients with CDI or CD colitis should be taken and that either no bowel prep or low-volume PEG solution should be preferred.

5.5.4.2. PPI use
A potential benefit of PPI use prior to upper GI FMT could reside in minimizing the effect of gastric acidity on altering engraftment of transplanted microorganisms [16, 67, 117]. PPIs might change microbiota [118] with moderate alterations occurring to both upper and distal gut microbiota [119]. No clinically significant benefit from routine use of PPIs in FMT protocols were identified $[120,121]$. Considering all these aspects, the WG suggested that PPI use should be avoided peri-procedurally, considering conflicting results on PPIs' effect in this setting, until further steps are taken to optimize protocols for safety and efficacy.

\subsubsection{Loperamide use}

A single dose/short course of loperamide was used for FMT recipients (predominantly for lower GI administration) with the purpose of extending exposure of the FMT to the mucosa, and to enhancing retention of the FMT within the GI tract [33, $67,72,81,109,121]$.

However, no in-depth analysis regarding these factors has been performed. Considering the pharmacokinetics of loperamide and the potential benefit in improving FMT retention, the WG agreed that a single-dose administered at least 2 hours before procedure, when FMT is delivered via lower GI and in the absence of severe colitis could be recommended, until further evidence is available.

5.5.4.4. Additional antibiotics pre-FMT and washout period between antibiotic use and FMT

Many studies on FMT included patients who had previously received conventional antimicrobial therapy, mainly with vancomycin or metronidazole [26, 28, 31]. One difference in various protocols of FMT for rCDI resides in the use of antibiotics, both the type of antibiotic and length of administration, ranging from 1 to 4 days prior to FMT $[122,123]$. Since it has been hypothesized that antibiotic prior to FMT might lead to a more effective engraftment of the donor microbiota to the recipient, generating a higher similarity between donor and recipient microbiota profiles after FMT and there are proof from preclinical studies that antibiotic treatment prior to FMT did not improve the overall engraftment of donor microbiota [124]. More direct evidence is required in order to generate specific protocol in this regard.

Furthermore, since antibiotic exposure prior and immediately after FMT has been identified as a predictor of FMT failure [67] a wash-out period of antibiotics should be considered prior to FMT infusion [104].

\subsubsection{Route of FMT delivery}

Administration of donor faeces can be performed via a nasogastric or nasoenteric tube, gastroduodenoscopy, colonoscopy, flexible sigmoidoscopy, enema or capsules. Although there is recent evidence that administering FMT using a nasogastric tube as route of administration is safe and effective [125] taking into account a recent meta-analysis evaluating the efficacy outcome of FMT depending on the route of administration suggested that colonoscopy is the most effective route, the WG supported using a colonoscopy as a first choice administration route whenever applicable, especially considering that FMT in Romania would be administered in credentialed tertiary centers, where colonoscopy is readily available. The same meta-analysis calculated cure rates were 
$78 \%$ identified and considering single infusion and $98 \%$ after overall infusions and identified that multiple infusions increased efficacy rates for each evaluated route of delivery (duodenal delivery: $73 \%$ with single infusion vs. $81 \%$ with multiple infusions; capsule: $80 \%$ vs. $92 \%$; colonoscopy: $78 \%$ vs. $98 \%$ and enema: $56 \%$ vs $92 \%$ ) [106]. However, it should be noted that the reported success rate when administering FMT by colonoscopy seems also dependent on delivering the transplant to the cecum. This may not be accessible in patients with severe colitis, in whom colonoscopy must be performed with caution, in order to minimize the risk of perforation, which makes consideration for upper GI route in these cases.

Although there is scarce data regarding FMT for treatment of $\mathrm{rCDI}$ in patients with stomas, the available reports on FMT delivered by upper endoscopy via an ileostomy was successful in treating severe complicated $\mathrm{rCDI}$, without noticeable adverse events [126].

It is recommended that all patients, healthcare providers and staff wear surgical facemasks at all times. FMT procedures involving mucous membranes including nasoenteric tube insertion, upper endoscopy or colonoscopy require N95 masks and face shields to be worn by personnel who perform the procedure and any members of the healthcare team present.

\subsubsection{Number of infusions}

At least 2 infusions should be considered in patients with rCDI showing signs of non-response persistent diarrhea at $48 \mathrm{~h}$ from first infusion. Despite having identified independent factors that may predict treatment failure after single infusion, as poor bowel preparation, endoscopic finding of pseudomembranes [68], and severe infection [32], there is a lack of validated criteria to guide the decision regarding the timing and number of infusions [127]. A recent meta-analysis confirmed that multiple stool infusions decreased with $76 \%$ the risk of rCDI [128]. Multiple infusion feces could significantly improve the effect of treatment. Ianiro et al. [36] found that a FMT protocol consisting of multiple fecal infusions and concomitant vancomycin was significantly more effective than a single FMT followed by vancomycin in curing severe CDI patients.

\section{BASIC REQUIREMENTS FOR IMPLEMENTING A FMT SERVICE}

There are different regulations for FMT among countries, and global regulation is lacking $[12,129]$. In the United Kingdom, the Medicines and Healthcare Products Regulatory Agency guides FMT practices, and an institute can conduct FMT legally after gaining permission from the agency [13]. The United States FDA initially considered FMT a drug, but the "enforcement discretion" regarding investigational new drug applications provided a possible means for FMT in patients with unresponsive or rCDI [130].

Authorities must regulate FMT to safeguard patients and donors, promote further research into safety and efficacy, and avoid abuse of the treatment. In 2012, the FDA classified human feces as a drug. However, The European Medicine Agency has not defined its position, leaving each country free to assign a qualification. In France, FMT is considered a drug by the
National Agency for Medicine and Health Product Safety, placing the fecal material under the jurisdiction of the hospital pharmacy [131]. Thus, its production should be carried out under the responsibility of pharmacists and storage within the pharmacy. Other European countries such as Austria or Finland see FMT as a therapeutic intervention that should not be considered a pharmaceutical drug and did not consider relevant to establish specific regulations. In those countries, doctors may perform FMT based on their own judgment without any approval from the drug authority [132].

Considering the potential side effects of FMT, this procedure should be authorized by the Ministry of Health and the credentialing and recredentialing process must involve the National Insurance House, the Ministry of Public Health, the Public Health Regulatory Council and the professional Societies: Romanian Society of Gastroenterology, Romanian Society of Microbiology.

In Romania, FMT will be performed under the regulation of the Ministry of Health, only in credentialed university centers, where infrastructure is available. We recommend that a multidisciplinary team should be responsible to complete FMT services. All adverse events and reactions related to FMT will be monitored and notified. Minimum infrastructure requirements should be fulfilled in order to achieve microbiological safety (outpatient room, sample FMT preparation and storage rooms with separate ventilation system and controlled environmenttemperature, humidity). Minimum equipment includes: $-80^{\circ} \mathrm{C}$ freezer, category 2 hood, clean but not sterile, equipped with scale, stomacher, and separate ventilation system. During FMT, we have to ensure the traceability of supply, monitoring, notification and investigation of all adverse events and reactions related to FMT. We recommend the creation of at least a local database (preferably a national database) including FMT recipients' data with careful monitoring of response to FMT and report adverse events, for a minimum 8-week follow-up, and a donor registry minimally including the date when donor screening process had begun and was completed and finally the date of the last completed screening process. Each center should establish a local clinical governance board, including at least a gastroenterologist, infectious disease specialist, microbiologist and epidemiologist.

Credentialing should include the evaluation of the performance indicators of the activity of the centre and the interval for recredentialing should be 3 years. The credentialing and recredentialing process must include the following competent institutions: The National Insurance House, the Ministry of Public Health, the Public Health Regulatory Council and professional Societies: Romanian Society of Gastroenterology, Romanian Society of Microbiology. It is essential to ensure the traceability of supply, using a quality system. All activities related to stool banking should rely on the principals of good clinical practice and follow the recommendations of the European Council Guide to the Quality and Safety of Tissues and Cells for Human Application [133].

\section{CONCLUSIONS}

Fecal microbiota transplantation is an effective treatment for patients with rCDI, being an established treatment in many countries. This report provides guidance on the 
general organization and the criteria required to perform FMT in patients with CDI. Despite sometimes with a low quality of evidence, a strong recommendation is recognized in considering the option of FMT for treatment of rCDI. This guideline should represent the scientific basis for FMT implementation in Romania.

\section{Conflicts of interest: None to declare.}

Authors' contribution: A.T., G.G., G.S. and I.G. participated in the design of the guideline. G.G., I.G. and C.M. collected and analyzed the data, drafted and revised the manuscript. A.T., T.I., J.S., B.M., N.Q., J.J.K., T.M., F.M., D.D., M.M., L.S.I., C.G., S.M. contributed to the analysis and interpretation of data. T.I., J.S., B.M., J.J.K., T.M. F.M., A.T. critically revised the manuscript. T.I., J.S., B.M., N.Q., J.J.K., T.M., R.M., D.D., M.M., L.S.I., C.G. and S.M. approved the final version of the final draft submitted. All authors have read and approved the final version submitted. All authors accept responsibility for its content.

Acknowledgements: The study was supported by UEG and is part of the activities included in a UEG Activity Grant. B.M. is the recipient of an NIHR Academic Clinical Lectureship, and is supported by the NIHR Imperial Biomedical Research Centre.

Supplementary material: To access the supplementary material visit the online version of the J Gastrointestin Liver Dis at http://dx.doi. org/10.15403/jgld-3297

\section{REFERENCES}

1. European Centre for Disease Prevention and Control. European Surveillance of Clostridioides (Clostridium) difficile infections: surveillance protocol version 2.4. 2019. Available at: https://www.ecdc. europa.eu/en/publications-data/european-surveillance-clostridiumdifficile-infections-surveillance-protocol-2

2. Davies KA, Ashwin H, Longshaw CM, et al. Diversity of Clostridium difficile PCR ribotypes in Europe: results from the European, multicentre, prospective, biannual, point-prevalence study of Clostridium difficile infection in hospitalised patients with diarrhoea (EUCLID), 2012 and 2013. Euro Surveill 2016;21(29). doi:10.2807/15607917.ES.2016.21.29.30294

3. Popescu GA, Serban R, Pistol A, et al. The Recent Emergence of Clostridium difficile Infection in Romanian Hospitals is Associated with a High Prevalence of Polymerase Chain Reaction Ribotype 027. Balkan Med J 2018;35:191-195. doi:10.4274/balkanmedj.2017.0400

4. Trifan A, Girleanu I, Stanciu C, et al. Clostridium difficile infection in hospitalized octogenarian patients: C. difficile infection in octogenarians. Geriatr Gerontol Int 2018;18:315-320. doi:10.1111/ggi.13186

5. Smits WK, Lyras D, Lacy DB, Wilcox MH, Kuijper EJ. Clostridium difficile infection. Nat Rev Dis Primers 2016;2:16020. doi:10.1038/ nrdp. 2016.20

6. Keller JJ, Kuijper EJ. Treatment of Recurrent and Severe Clostridium Difficile Infection. Annu Rev Med 2015;66:373-386. doi:10.1146/ annurev-med-070813-114317

7. Burton HE, Mitchell SA, Watt M. A Systematic Literature Review of Economic Evaluations of Antibiotic Treatments for Clostridium difficile Infection. Pharmacoeconomics 2017;35:1123-1140. doi:10.1007/ s40273-017-0540-2
8. Quraishi MN, Widlak M, Bhala N, et al. Systematic review with meta-analysis: the efficacy of faecal microbiota transplantation for the treatment of recurrent and refractory Clostridium difficile infection. Aliment Pharmacol Ther 2017;46:479-493. doi:10.1111/apt.14201

9. Preda CM, Meianu C, Sandra I, et al. Fecal Microbiota Transplantation in Recurrent NAP1/B1/027 Clostridium Difficile Infection (CDI) Resistant to Vancomycin and Metronidazole in a Patient with Ulcerative Colitis (UC): A Case Report. Rev Med Chir Soc Med Nat Iasi 2016;120:563567.

10. Mihaela L, Pascu O, Leucuta DC, Andreica V. Fecal microbiota transplantation in recurrent Clostridium difficile infection: the first prospective study of 30 patients in Romania. Rev Romana Med Lab 2018;26:201-210. doi:10.2478/rrlm-2018-0013

11. Madar PC, Petre O, Baban A, Dumitrascu DL. Medical students perception on fecal microbiota transplantation. BMC Med Educ 2019;19:368. doi:10.1186/s12909-019-1804-7

12. Cammarota G, Ianiro G, Tilg H, et al. European consensus conference on faecal microbiota transplantation in clinical practice. Gut 2017;66:569580. doi:10.1136/gutjnl-2016-313017

13. Mullish BH, Quraishi MN, Segal JP, et al. The use of faecal microbiota transplant as treatment for recurrent or refractory Clostridium difficile infection and other potential indications: joint British Society of Gastroenterology (BSG) and Healthcare Infection Society (HIS) guidelines. Gut 2018;67:1920-1941. doi:10.1136/gutjnl-2018-316818

14. Keller JJ, Ooijevaar RE, Hvas CL, et al. A standardised model for stool banking for faecal microbiota transplantation: a consensus report from a multidisciplinary UEG working group. United European Gastroenterol J 2020;205064062096789. doi:10.1177/2050640620967898

15. Ianiro G, Mullish BH, Kelly CR, et al. Screening of faecal microbiota transplant donors during the COVID-19 outbreak: suggestions for urgent updates from an international expert panel. Lancet Gastroenterol Hepatol 2020;5:430-432. doi:10.1016/S2468-1253(20)30082-0

16. Cohen SH, Gerding DN, Johnson S, et al. Clinical Practice Guidelines for Clostridium difficile Infection in Adults: 2010 Update by the Society for Healthcare Epidemiology of America (SHEA) and the Infectious Diseases Society of America (IDSA). Infect Control Hosp Epidemiol 2010;31:431-455. doi:10.1086/651706

17. Rao K, Malani PN. Diagnosis and Treatment of Clostridioides (Clostridium) difficile Infection in Adults in 2020. JAMA 2020;323:1403. doi:10.1001/jama.2019.3849

18. Cheng YW, Fischer M. Fecal Microbiota Transplantation: Redefining Surgical Management of Refractory Clostridium difficile Infection. Clin Colon Rectal Surg 2020;33:92-97. doi:10.1055/s-0040-1701233

19. Surawicz CM, Brandt LJ, Binion DG, et al. Guidelines for Diagnosis, Treatment, and Prevention of Clostridium difficile Infections. Am J Gastroenterol 2013;108:478-498. doi:10.1038/ajg.2013.4

20. Debast SB, Bauer MP, Kuijper EJ; European Society of Clinical Microbiology and Infectious Diseases. European Society of Clinical Microbiology and Infectious Diseases: Update of the Treatment Guidance Document for Clostridium difficile Infection. Clin Microbiol Infect 2014;20 Suppl 2:1-26. doi:10.1111/1469-0691.12418

21. Lee CH, Steiner T, Petrof EO, et al. Frozen vs Fresh Fecal Microbiota Transplantation and Clinical Resolution of Diarrhea in Patients with Recurrent Clostridium difficile Infection: A Randomized Clinical Trial. JAMA 2016;315:142-149. doi:10.1001/jama.2015.18098

22. Hoffmann DE, Palumbo FB, Ravel J, Rowthorn V, von Rosenvinge E. A proposed definition of microbiota transplantation for regulatory purposes. Gut Microbes 2017;8:208-213. doi:10.1080/19490976.2017. 1293223 
23. Cammarota G, Ianiro G, Kelly CR, et al. International consensus conference on stool banking for faecal microbiota transplantation in clinical practice. Gut 2019;68:2111-2121. doi:10.1136/ gutjnl-2019-319548

24. Guyatt GH, Oxman AD, Vist GE, et al. GRADE: an emerging consensus on rating quality of evidence and strength of recommendations. BM] 2008;336:924-926. doi:10.1136/bmj.39489.470347.AD

25. Cammarota G, Masucci L, Ianiro G, et al. Randomised clinical trial: faecal microbiota transplantation by colonoscopy vs. vancomycin for the treatment of recurrent Clostridium difficile infection. Aliment Pharmacol Ther 2015;41:835-843. doi:10.1111/apt.13144

26. Kelly CR, Khoruts A, Staley C, et al. Effect of Fecal Microbiota Transplantation on Recurrence in Multiply Recurrent Clostridium difficile Infection: A Randomized Trial. Ann Intern Med 2016;165:609616. doi:10.7326/M16-0271

27. van Nood E, Vrieze A, Nieuwdorp M, et al. Duodenal Infusion of Donor Feces for Recurrent Clostridium difficile. N Engl J Med 2013;368:407_ 415. doi:10.1056/NEJMoa1205037

28. Hota SS, Sales V, Tomlinson G, et al. Oral Vancomycin Followed by Fecal Transplantation Versus Tapering Oral Vancomycin Treatment for Recurrent Clostridium difficile Infection: An Open-Label, Randomized Controlled Trial. Clin Infect Dis 2017;64:265-271. doi:10.1093/cid/ ciw731

29. Dubberke ER, Lee CH, Orenstein R, Khanna S, Hecht G, Gerding DN. Results From a Randomized, Placebo-Controlled Clinical Trial of a RBX2660-A Microbiota-Based Drug for the Prevention of Recurrent Clostridium difficile Infection. Clin Infect Dis 2018;67:1198-1204 doi:10.1093/cid/ciy259

30. Hvas CL, Dahl Jørgensen SM, Jørgensen SP, et al. Fecal Microbiota Transplantation Is Superior to Fidaxomicin for Treatment of Recurrent Clostridium difficile Infection. Gastroenterology 2019;156:1324-1332. e3. doi:10.1053/j.gastro.2018.12.019

31. Cammarota G, Masucci L, Ianiro G, et al. Randomised clinical trial: faecal microbiota transplantation by colonoscopy vs. vancomycin for the treatment of recurrent Clostridium difficile infection. Aliment Pharmacol Ther 2015;41:835-843. doi:10.1111/apt.13144

32. Fischer M, Kao D, Mehta SR, et al. Predictors of Early Failure After Fecal Microbiota Transplantation for the Therapy of Infection: A Multicenter Study. Am J Gastroenterol 2016;111:1024-1031. doi:10.1038/ajg.2016.180

33. Agrawal M, Aroniadis OC, Brandt LJ, et al. The Long-term Efficacy and Safety of Fecal Microbiota Transplant for Recurrent, Severe, and Complicated Clostridium difficile Infection in 146 Elderly Individuals. J Clin Gastroenterol 2019;50:403-407. doi:10.1097/ MCG.0000000000000410

34. Alukal J, Dutta SK, Surapaneni BK, et al. Safety and efficacy of fecal microbiota transplant in 9 critically ill patients with severe and complicated Clostridium difficile infection with impending colectomy. J Dig Dis 2019;20:301-307. doi:10.1111/1751-2980.12750

35. Hocquart M, Lagier JC, Cassir N, et al. Early Fecal Microbiota Transplantation Improves Survival in Severe Clostridium difficile Infections. Clin Infect Dis 2018;66:645-650. doi:10.1093/cid/cix762

36. Ianiro G, Masucci L, Quaranta G, et al. Randomised clinical trial: faecal microbiota transplantation by colonoscopy plus vancomycin for the treatment of severe refractory Clostridium difficile infection-single versus multiple infusions. Aliment Pharmacol Ther 20181;48:152-159. doi:10.1111/apt.14816

37. Cheng YW, Phelps E, Nemes S, et al. Fecal Microbiota Transplant Decreases Mortality in Patients with Refractory Severe or Fulminant
Clostridioides difficile Infection. Clin Gastroenterol Hepatol 2020;18:2234-2243.e1. doi:10.1016/j.cgh.2019.12.029

38. van Beurden YH, Nieuwdorp M, van de Berg PJEJ, Mulder CJJ, Goorhuis A. Current challenges in the treatment of severe Clostridium difficile infection: early treatment potential of fecal microbiota transplantation. Therap Adv Gastroenterol 2017;10:373-381. doi:10.1177/1756283X17690480

39. Falcone M, Tiseo G, Iraci F, et al. Risk factors for recurrence in patients with Clostridium difficile infection due to 027 and non-027 ribotypes. Clin Microbiol Infect 2019;25:474-480. doi:10.1016/j.cmi.2018.06.020

40. Song JH, Kim YS. Recurrent Clostridium difficile Infection: Risk Factors, Treatment, and Prevention. Gut Liver 2019;13:16-24. doi:10.5009/ gnl18071

41. Paramsothy S, Kamm MA, Kaakoush NO, et al. Multidonor intensive faecal microbiota transplantation for active ulcerative colitis: a randomised placebo-controlled trial. Lancet 2017;389:1218-1228. doi:10.1016/S0140-6736(17)30182-4

42. Costello SP, Hughes PA, Waters O, et al. Effect of Fecal Microbiota Transplantation on 8-Week Remission in Patients With Ulcerative Colitis: A Randomized Clinical Trial. JAMA 2019;321:156-164. doi:10.1001/jama.2018.20046

43. Rossen NG, Fuentes S, van der Spek MJ, et al. Findings From a Randomized Controlled Trial of Fecal Transplantation for Patients With Ulcerative Colitis. Gastroenterology 2015;149:110-118.e4. doi:10.1053/j. gastro.2015.03.045

44. Sokol H, Galperine T, Kapel N, et al. Faecal microbiota transplantation in recurrent Clostridium difficile infection: Recommendations from the French Group of Faecal microbiota Transplantation. Dig Liver Dis 2016;48:242-247. doi:10.1016/j.dld.2015.08.017

45. Aroniadis OC, Brandt LJ, Oneto C, et al. Faecal microbiota transplantation for diarrhoea-predominant irritable bowel syndrome: a double-blind, randomised, placebo-controlled trial. Lancet Gastroenterol Hepatol 2019;4:675-685. doi:10.1016/S2468-1253(19)30198-0

46. Halkjær SI, Christensen AH, Lo BZS, et al. Faecal microbiota transplantation alters gut microbiota in patients with irritable bowel syndrome: results from a randomised, double-blind placebo-controlled study. Gut 2018;67:2107-2115. doi:10.1136/gutjnl-2018-316434

47. Johnsen PH, Hilpüsch F, Cavanagh JP, et al. Faecal microbiota transplantation versus placebo for moderate-to-severe irritable bowel syndrome: a double-blind, randomised, placebo-controlled, parallelgroup, single-centre trial. Lancet Gastroenterol Hepatol 2018;3:17-24 doi:10.1016/S2468-1253(17)30338-2

48. Holster S, Lindqvist CM, Repsilber D, et al. The Effect of Allogenic Versus Autologous Fecal Microbiota Transfer on Symptoms, Visceral Perception and Fecal and Mucosal Microbiota in Irritable Bowel Syndrome: A Randomized Controlled Study. Clin Transl Gastroenterol 2019;10:e00034. doi:10.14309/ctg.0000000000000034

49. El-Salhy M, Hatlebakk JG, Gilja OH, Bråthen Kristoffersen A, Hausken T. Efficacy of faecal microbiota transplantation for patients with irritable bowel syndrome in a randomised, double-blind, placebo-controlled study. Gut 2020;69:859-867. doi:10.7326/ACPJ202005190-052

50. Lahtinen P, Jalanka J, Hartikainen A, et al. Randomised clinica trial: faecal microbiota transplantation versus autologous placebo administered via colonoscopy in irritable bowel syndrome. Aliment Pharmacol Ther 2020;51:1321-1331. doi:10.1111/apt.15740

51. Bajaj JS, Kassam Z, Fagan A, et al. Fecal microbiota transplant from a rational stool donor improves hepatic encephalopathy: A randomized clinical trial Hepatology 2017;66:1727-1738. doi:10.1002/hep.29306 
52. Huttner BD, de Lastours V, Wassenberg M, et al. A 5-day course of ora antibiotics followed by faecal transplantation to eradicate carriage of multidrug-resistant Enterobacteriaceae: a randomized clinical trial. Clin Microbiol Infect 2019;25:830-838. doi:10.1016/j.cmi.2018.12.009

53. Louie TJ, Miller MA, Mullane KM, et al. Fidaxomicin versus Vancomycin for Clostridium difficile Infection. N Engl J Med 2011;364:422-431. doi:10.1056/NEJMoa0910812

54. Wilcox MH, Gerding DN, Poxton IR, et al. Bezlotoxumab for Prevention of Recurrent Clostridium difficile Infection. N Engl J Med 2017;376:305-317. doi:10.1056/NEJMoa1602615

55. Borody TJ, Khoruts A. Fecal microbiota transplantation and emerging applications. Nat Rev Gastroenterol Hepatol 2012;9:88-96. doi:10.1038/ nrgastro.2011.244

56. Weingarden AR, Hamilton MJ, Sadowsky MJ, Khoruts A. Resolution of Severe Clostridium difficile Infection Following Sequential Fecal Microbiota Transplantation. J Clin Gastroenterol 2013;47:735-737. doi:10.1097/MCG.0b013e31829004ae

57. Tariq R, Disbrow MB, Dibaise JK, et al. Efficacy of Fecal Microbiota Transplantation for Recurrent C. Difficile Infection in Inflammatory Bowel Disease. Inflamm Bowel Dis 2020;26:1415-1420. doi:10.1093/ $\mathrm{ibd} / \mathrm{izz} 299$

58. DeFilipp Z, Bloom PP, Torres Soto M, et al. Drug-Resistant E. coli Bacteremia Transmitted by Fecal Microbiota Transplant. N Engl J Med 2019;381:2043-2050. doi:10.1056/NEJMoa1910437

59. Food and Drug Administration. Important Safety Alert Regarding Use of Fecal Microbiota for Transplantation and Risk of Serious Adverse Reactions Due to Transmission of Multi-Drug Resistant Organisms. Available at: https://www.fda.gov/vaccines-blood-biologics/safetyavailability-biologics/important-safety-alert-regarding-use-fecalmicrobiota-transplantation-and-risk-serious-adverse

60. Kelly CR, Ihunnah C, Fischer M, et al. Fecal Microbiota Transplant for Treatment of Clostridium difficile Infection in Immunocompromised Patients. Am J Gastroenterol 2014;109:1065-1071. doi:10.1038/ ajg. 2014.133

61. Saeedi BJ, Morison DG, Kraft CS, Dhere T. Fecal Microbiota Transplant for Clostridium difficile Infection in a Pregnant Patient. Obstet Gynecol 2017;129:507-509. doi:10.1097/AOG.0000000000001911

62. Tariq R, Pardi DS, Bartlett MG, Khanna S. Low Cure Rates in Controlled Trials of Fecal Microbiota Transplantation for Recurrent Clostridium difficile Infection: A Systematic Review and Meta-analysis. Clin Infect Dis 2019;68:1351-1358. doi:10.1093/cid/ciy721

63. Kelly CR, de Leon L, Jasutkar N. Fecal Microbiota Transplantation for Relapsing Clostridium difficile Infection in 26 Patients: Methodology and Results. J Clin Gastroenterol 2012;46:145-149. doi:10.1097/ MCG.0b013e318234570b

64. Aroniadis OC, Brandt LJ, Greenberg A, et al. Long-term Follow-up Study of Fecal Microbiota Transplantation for Severe and/or Complicated Clostridium difficile Infection: A Multicenter Experience. J Clin Gastroenterol 2016;50:398-402. doi:10.1097/MCG.0000000000000374

65. Sokol H, Galperine T, Kapel N, et al. Faecal microbiota transplantation in recurrent Clostridium difficile infection: Recommendations from the French Group of Faecal microbiota Transplantation. Dig Liver Dis 2016;48:242-247. doi:10.1016/j.dld.2015.08.017

66. Allegretti JR, Allegretti AS, Phelps E, Xu H, Fischer M, Kassam Z. Classifying Fecal Microbiota Transplantation Failure: An Observational Study Examining Timing and Characteristics of Fecal Microbiota Transplantation Failures. Clin Gastroenterol Hepatol 2018;16:18321833. doi:10.1016/j.cgh.2017.10.031
67. Alrabaa S, Jariwala R, Zeitler K, Montero J. Fecal microbiota transplantation outcomes in immunocompetent and immunocompromised patients: A single-center experience. Transpl Infect Dis 2017;19:e12726. doi:10.1111/ tid. 12726

68. Ianiro G, Valerio L, Masucci L, et al. Predictors of failure after single faecal microbiota transplantation in patients with recurrent Clostridium difficile infection: results from a 3-year, singlecentre cohort study. Clin Microbiol Infect 2017;23:337.e1-337.e3. doi:10.1016/j.cmi.2016.12.025

69. Terveer EM, Vendrik KE, Ooijevaar RE, et al. Faecal microbiota transplantation for Clostridioides difficile infection: Four years' experience of the Netherlands Donor Feces Bank. United European Gastroenterol J 2020;8:1236-1247. doi:10.1177/2050640620957765

70. Rokkas T, Gisbert JP, Gasbarrini A, et al. A network metaanalysis of randomized controlled trials exploring the role of fecal microbiota transplantation in recurrent Clostridium difficile infection. United European Gastroenterol J 2019;7:1051-1063. doi:10.1177/2050640619854587

71. Hamilton MJ, Weingarden AR, Sadowsky MJ, Khoruts A. Standardized Frozen Preparation for Transplantation of Fecal Microbiota for Recurrent Clostridium difficile Infection. Am J Gastroenterol 2012;107:761-767. doi:10.1038/ajg.2011.482

72. Ray A, Smith R, Breaux J. Fecal Microbiota Transplantation for Clostridium difficile Infection: The Ochsner Experience. Ochsner J 2014;14:538-544.

73. MacConnachie AA, Fox R, Kennedy DR, Seaton RA. Faecal transplant for recurrent Clostridium difficile-associated diarrhoea: a UK case series. QJM 2009;102:781-784. doi:10.1093/qjmed/hcp118

74. Newman KM, Rank KM, Vaughn BP, Khoruts A. Treatment of recurrent Clostridium difficile infection using fecal microbiota transplantation in patients with inflammatory bowel disease. Gut Microbes 2017;8:303309. doi:10.1080/19490976.2017.1279377

75. Kociolek LK, Gerding DN. Breakthroughs in the treatment and prevention of Clostridium difficile infection. Nat Rev Gastroenterol Hepatol 2016;13:150-160. doi:10.1038/nrgastro.2015.220

76. Kassam Z, Hundal R, Marshall JK, Lee CH. Fecal Transplant via Retention Enema for Refractory or Recurrent Clostridium difficile Infection. Arch Intern Med 2012;172:191-193. doi:10.1001/ archinte.172.2.191

77. Baxter M, Colville A. Adverse events in faecal microbiota transplant: a review of the literature. J Hosp Infect 2016;92:117-127. doi:10.1016/j. jhin.2015.10.024

78. Schwartz M, Gluck M, Koon S. Norovirus Gastroenteritis After Fecal Microbiota Transplantation for Treatment of Clostridium difficile Infection Despite Asymptomatic Donors and Lack of Sick Contacts. Am J Gastroenterol 2013;108:1367-1367. doi:10.1038/ajg.2013.164

79. Food and Drug Administration. Fecal Microbiota for Transplantation: Safety Alert - Risk of Serious Adverse Events Likely Due to Transmission of Pathogenic Organisms. Available at: https://www.fda.gov/safety/ medical-product-safety-information/fecal-microbiota-transplantationsafety-alert-risk-serious-adverse-events-likely-due-transmission

80. Wang S, Xu M, Wang W, et al. Systematic Review: Adverse Events of Fecal Microbiota Transplantation. PLoS One 2016;11:e0161174. doi:10.1371/journal.pone.0161174

81. Patel NC, Griesbach CL, DiBaise JK, Orenstein R. Fecal Microbiota Transplant for Recurrent Clostridium difficile Infection: Mayo Clinic in Arizona Experience. Mayo Clin Proc 2013;88:799-805. doi:10.1016/j. mayocp.2013.04.022 
82. Allegretti JR, Kassam Z, Osman M, Budree S, Fischer M, Kelly CR. The 5D framework: a clinical primer for fecal microbiota transplantation to treat Clostridium difficile infection. Gastrointest Endosc 2018;87:18-29. doi:10.1016/j.gie.2017.05.036

83. Ianiro G, Mullish BH, Kelly CR, et al. Reorganisation of faecal microbiota transplant services during the COVID-19 pandemic. Gut 2020;69:1555-1563. doi:10.1136/gutjnl-2020-321829

84. Brandt LJ, Aroniadis OC, Mellow M, et al. Long-Term Follow-Up of Colonoscopic Fecal Microbiota Transplant for Recurrent Clostridium difficile Infection. Am J Gastroenterol 2012;107:1079-1087. doi:10.1038/ajg.2012.60

85. Shogbesan O, Poudel DR, Victor S, et al. A Systematic Review of the Efficacy and Safety of Fecal Microbiota Transplant for Clostridium difficile Infection in Immunocompromised Patients. Can J Gastroenterol Hepatol 2018;2018:1394379. doi:10.1155/2018/1394379

86. Wardill HR, Secombe KR, Bryant RV, Hazenberg MD, Costello SP. Adjunctive fecal microbiota transplantation in supportive oncology: Emerging indications and considerations in immunocompromised patients. EBioMedicine 2019;44:730-740. doi:10.1016/j. ebiom.2019.03.070

87. Ramay FH, Amoroso A, von Rosenvinge EC, Saharia K. Fecal Microbiota Transplantation for Treatment of Severe, Recurrent, and Refractory Clostridium difficile Infection in a Severely Immunocompromised Patient. Infect Dis Clin Pract 2015;24:237-240. doi:10.1097/ IPC.0000000000000338

88. Stripling J, Kumar R, Baddley JW, et al. Loss of Vancomycin-Resistant Enterococcus Fecal Dominance in an Organ Transplant Patient With Clostridium difficile Colitis After Fecal Microbiota Transplant. Open Forum Infect Dis 2015;2:ofv078. doi:10.1093/ofid/ofv078

89. Cheng YW, Phelps E, Ganapini V, et al. Fecal microbiota transplantation for the treatment of recurrent and severe Clostridium difficile infection in solid organ transplant recipients: A multicenter experience. Am J Transplant 2019;19:501-511. doi:10.1111/ajt.15058

90. Bajaj JS, Salzman NH, Acharya C, et al. Fecal Microbial Transplant Capsules Are Safe in Hepatic Encephalopathy: A Phase 1, Randomized, Placebo-Controlled Trial. Hepatology 2019;70:1690-1703. doi:10.1002/ hep.30690

91. Meighani A, Alimirah M, Ramesh M, Salgia R. Fecal Microbiota Transplantation for Clostridioides Difficile Infection in Patients with Chronic Liver Disease. Int J Hepatol 2020;2020:1874570. doi:10.1155/2020/1874570

92. Dai M, Liu Y, Chen W, et al. Rescue fecal microbiota transplantation for antibiotic-associated diarrhea in critically ill patients. Crit Care 2019;23:324. doi:10.1186/s13054-019-2604-5

93. Tixier EN, Verheyen E, Ungaro RC, Grinspan AM. Faecal microbiota transplant decreases mortality in severe and fulminant Clostridioides difficile infection in critically ill patients. Aliment Pharmacol Ther 2019;50:1094-1099. doi:10.1111/apt.15526

94. Trubiano JA, Gardiner B, Kwong JC, Ward P, Testro AG, Charles PGP. Faecal microbiota transplantation for severe Clostridium difficile infection in the intensive care unit. Eur J Gastroenterol Hepatol 2013;25:255-257. doi:10.1097/MEG.0b013e32835b2da9

95. Bakken JS, Borody T, Brandt LJ, et al. Treating Clostridium difficile Infection With Fecal Microbiota Transplantation. Clin Gastroenterol Hepatol 2011;9:1044-1049. doi:10.1016/j.cgh.2011.08.014

96. Youngster I, Sauk J, Pindar C, et al. Fecal Microbiota Transplant for Relapsing Clostridium difficile Infection Using a Frozen Inoculum From Unrelated Donors: A Randomized, Open-Label, Controlled Pilot Study. Clin Infect Dis 2014;58:1515-1522. doi:10.1093/cid/ciu135
97. Kassam Z, Lee CH, Yuan Y, Hunt RH. Fecal Microbiota Transplantation for Clostridium difficile Infection: Systematic Review and MetaAnalysis. Am J Gastroenterol 2013;108:500-508. doi:10.1038/ ajg.2013.59

98. Weingarden AR, Chen C, Bobr A, et al. Microbiota transplantation restores normal fecal bile acid composition in recurrent Clostridium difficile infection. Am J Physiol Gastrointest Liver Physiol 2014;306:G310-G319. doi:10.1152/ajpgi.00282.2013

99. Shankar V, Hamilton MJ, Khoruts A, et al. Species and genus level resolution analysis of gut microbiota in Clostridium difficile patients following fecal microbiota transplantation. Microbiome 2014;2:13. doi:10.1186/2049-2618-2-13

100. Costello SP, Tucker EC, La Brooy J, Schoeman MN, Andrews JM. Establishing a Fecal Microbiota Transplant Service for the Treatment of Clostridium difficile Infection. Clin Infect Dis 2016;62:908-914. doi:10.1093/cid/civ994

101. Claesson MJ, Cusack S, O’Sullivan O, et al. Composition, variability, and temporal stability of the intestinal microbiota of the elderly. Proc Natl Acad Sci U S A 2011;108 (Suppl 1):4586-4591. doi:10.1073/ pnas. 1000097107

102. García-Peña C, Álvarez-Cisneros T, Quiroz-Baez R, Friedland RP. Microbiota and Aging. A Review and Commentary. Arch Med Res 2017;48:681-689. doi:10.1016/j.arcmed.2017.11.005

103. Alang N, Kelly CR. Weight Gain After Fecal Microbiota Transplantation. Open Forum Infect Dis 2015;2:ofv004. doi:10.1093/ofid/ofv004

104. Fischer M, Kao D, Kassam Z, et al. Stool Donor Body Mass Index Does Not Affect Recipient Weight After a Single Fecal Microbiota Transplantation for Clostridium difficile Infection. Clin Gastroenterol Hepatol 2018;16:1351-1353. doi:10.1016/j.cgh.2017.12.007

105. Food and Drug Administration. Safety Alert Regarding Use of Fecal Microbiota for Transplantation and Additional Safety Protections Pertaining to SARS-CoV-2 and COVID-19. Available at: https://www. fda.gov/vaccines-blood-biologics/safety-availability-biologics/safetyalert-regarding-use-fecal-microbiota-transplantation-and-additionalsafety-protections

106. Ianiro G, Maida M, Burisch J, et al. Efficacy of different faecal microbiota transplantation protocols for Clostridium difficile infection: A systematic review and meta-analysis. United European Gastroenterol J 2018;6:1232-1244. doi:10.1177/2050640618780762

107. Lynch SM, Mu J, Grady JJ, Stevens RG, Devers TJ. Fecal Microbiota Transplantation for Clostridium difficile Infection: A One-Center Experience. Dig Dis 2019;37:467-472. doi:10.1159/000499873

108. Youngster I, Mahabamunuge J, Systrom HK, et al. Oral, frozen fecal microbiota transplant (FMT) capsules for recurrent Clostridium difficile infection. BMC Med. 2016;14:134. doi:10.1186/s12916-016-0680-9

109. Jiang ZD, Ajami NJ, Petrosino JF, et al. Randomised clinical trial: faecal microbiota transplantation for recurrent Clostridum difficile infection - fresh, or frozen, or lyophilised microbiota from a small pool of healthy donors delivered by colonoscopy. Aliment Pharmacol Ther 2017;45:899-908. doi:10.1111/apt.13969

110. O’Brien CL, Allison GE, Grimpen F, Pavli P. Impact of Colonoscopy Bowel Preparation on Intestinal Microbiota. PLoS One 2018;8:e62815. doi:10.1371/journal.pone.0062815

111. Mai V, Stine OC. Bowel preparation for colonoscopy: relevant for the gut's microbiota? Gut 2015;64:1504-1505. doi:10.1136/ gutjnl-2014-308937

112. Jalanka J, Salonen A, Salojärvi J, et al. Effects of bowel cleansing on the intestinal microbiota. Gut 2015;64:1562-1568. doi:10.1136/ gutjnl-2014-307240 
113. Persky SE, Brandt LJ. Treatment of recurrent Clostridium difficileassociated diarrhea by administration of donated stool directly through a colonoscope. Am J Gastroenterol 2000;95:3283-3285. doi:10.1111/ j.1572-0241.2000.03302.x

114. Terveer EM, van Beurden YH, Goorhuis A, et al. How to: Establish and run a stool bank. Clin Microbiol Infect 2017;23:924-930. doi:10.1016/j. cmi.2017.05.015

115. Jiang Z-D, Jenq RR, Ajami NJ, et al. Safety and preliminary efficacy of orally administered lyophilized fecal microbiota product compared with frozen product given by enema for recurrent Clostridium difficile infection: A randomized clinical trial. PLoS One 2018;13:e0205064. doi:10.1371/journal.pone.0205064

116. Restellini S, Kherad O, Bessissow T, et al. Systematic review and metaanalysis of colon cleansing preparations in patients with inflammatory bowel disease. World J Gastroenterol 2017;23:5994-6002. doi:10.3748/ wjg.v23.i32.5994

117. Zainah H, Hassan M, Shiekh-Sroujieh L, Hassan S, Alangaden G, Ramesh M. Intestinal Microbiota Transplantation, a Simple and Effective Treatment for Severe and Refractory Clostridium Difficile Infection. Dig Dis Sci 2015;60:181-185. doi:10.1007/s10620-014-3296-y

118. Naito Y, Kashiwagi K, Takagi T, Andoh A, Inoue R. Intestinal Dysbiosis Secondary to Proton-Pump Inhibitor Use. Digestion 2018;97:195-204. doi: $10.1159 / 000481813$

119. Macke L, Schulz C, Koletzko L, Malfertheiner P. Systematic review: the effects of proton pump inhibitors on the microbiome of the digestive tract-evidence from next-generation sequencing studies. Aliment Pharmacol Ther 2020;51:505-526. doi:10.1111/apt.15604

120. Hong AS, Yu WY, Hong JM, et al. Proton pump inhibitor in upper gastrointestinal fecal microbiota transplant: A systematic review and analysis. J Gastroenterol Hepatol 2020;35:932-940. doi:10.1111/ jgh. 14958

121. Peri R, Aguilar RC, Tüffers K, et al. The impact of technical and clinical factors on fecal microbiota transfer outcomes for the treatment of recurrent Clostridioides difficile infections in Germany. United European Gastroenterol J 2019;7:716-722. doi:10.1177/2050640619839918

122. Pathak R, Enuh HA, Patel A, Wickremesinghe P. Treatment of relapsing Clostridium : difficile infection using fecal microbiota transplantation. Clin Exp Gastroenterol 2013;7:1-6. doi:10.2147/CEG.S53410

123. Rubin TA, Gessert CE, Aas J, Bakken JS. Fecal microbiome transplantation for recurrent Clostridium difficile infection: Report on a case series. Anaerobe 2013;19:22-26. doi:10.1016/j. anaerobe.2012.11.004
124. Freitag TL, Hartikainen A, Jouhten H, et al. Minor Effect of Antibiotic Pre-treatment on the Engraftment of Donor Microbiota in Fecal Transplantation in Mice. Front Microbiol 2019;10:2685. doi:10.3389/ fmicb.2019.02685

125. McCune VL, Quraishi MN, Manzoor S, et al. Results from the first English stool bank using faecal microbiota transplant as a medicinal product for the treatment of Clostridioides difficile infection. EClinicalMedicine 2020;20:100301. doi:10.1016/j.eclinm.2020.100301

126. Digby-Bell J, Williams A, Irving P, Goldenberg S. Successful faeca microbiota transplant for recurrent Clostridium difficile infection delivered by colonoscopy through a diverted ileostomy in a patient with severe perianal Crohn's disease. BMJ Case Rep 2018;2018: bcr2017222958. doi:10.1136/bcr-2017-222958

127. Gallo A, Cancelli C, Ceron E, et al. Fecal calprotectin and need of multiple microbiota trasplantation infusions in Clostridium difficile infection. J Gastroenterol Hepatol 2020;35:1909-1915. doi:10.1111/ jgh. 15072

128. Hui W, Li T, Liu W, Zhou C, Gao F. Fecal microbiota transplantation for treatment of recurrent C. difficile infection: An updated randomized controlled trial meta-analysis. PLoS One 2019;14:e0210016. doi:10.1371/journal.pone.0210016

129. Kelly CR, Kahn S, Kashyap P, et al. Update on Fecal Microbiota Transplantation 2015: Indications, Methodologies, Mechanisms, and Outlook. Gastroenterology 2015;149:223-237. doi:10.1053/j. gastro.2015.05.008

130. Kelly CR, Kim AM, Laine L, Wu GD. The AGA's Fecal Microbiota Transplantation National Registry: An Important Step Toward Understanding Risks and Benefits of Microbiota Therapeutics. Gastroenterology 2017;152:681-684. doi:10.1053/j. gastro.2017.01.028

131. Kelly CR, Laine LA, Wu GD. Monitoring fecal microbiota transplantation practice in a rapidly evolving health and regulatory environment. Gastroenterology 2020;159:2004-2006. doi:10.1053/j.gastro.2020.08.039

132. Schneider A, Maric L. Fecal Microbiota Transplantation as Therapy for Inflammatory Bowel Disease. In: Schen B. (ed.). Interventional Inflammatory Bowel Disease: Endoscopic Management and Treatment of Complications. Elsevier; 2018:319-327. doi:10.1016/C2016-0-015085

133. Council of Europe. New Guide to the quality and safety of tissues and cells for human application. $4^{\text {th }}$ edition. Available at: https://www.edqm. $\mathrm{eu} / \mathrm{en} / \mathrm{news} / \mathrm{new}$-guide-quality-and-safety-tissues-and-cells-humanapplication 